\title{
TIME CONSTRAINTS MEDIATE PREDATOR-INDUCED PLASTICITY IN IMMUNE FUNCTION, CONDITION, AND LIFE HISTORY
}

\author{
Robby Stoks, ${ }^{1}$ Marjan De Block,,${ }^{1,2}$ Stefanie Slos, ${ }^{1}$ Wendy Van Doorslaer, ${ }^{1}$ and Jens RolfF 3,4 \\ ${ }^{1}$ Laboratory of Aquatic Ecology, University of Leuven, Ch. De Bériotstraat 32, B-3000 Leuven, Belgium \\ ${ }^{2}$ Department of Biological Sciences, Dartmouth College, Hanover, New Hampshire 03755 USA \\ ${ }^{3}$ Department of Animal and Plant Sciences, University of Sheffield S10 2TN, Sheffield, UK
}

\begin{abstract}
The simultaneous presence of predators and a limited time for development imposes a conflict: accelerating growth under time constraints comes at the cost of higher predation risk mediated by increased foraging. The few studies that have addressed this tradeoff have dealt only with life history traits such as age and size at maturity. Physiological traits have largely been ignored in studies assessing the impact of environmental stressors, and it is largely unknown whether they respond independently of life history traits. Here, we studied the simultaneous effects of time constraints, i.e., as imposed by seasonality, and predation risk on immune defense, energy storage, and life history in lestid damselflies. As predicted by theory, larvae accelerated growth and development under time constraints while the opposite occurred under predation risk. The activity of phenoloxidase, an important component of insect immunity, and investment in fat storage were reduced both under time constraints and in the presence of predators. These reductions were smaller when time constraints and predation risk were combined. This indicates that predators can induce sublethal costs linked to both life history and physiology in their prey, and that time constraints can independently reduce the impact of predator-induced changes in life history and physiology.
\end{abstract}

Key words: carry-over costs; complex life cycle; damselfy larvae; fat reserves; immune function; life history plasticity; predation risk; time constraints.

\section{INTRODUCTION}

Complex life cycles that comprise discrete stages with contrasting behavioral, morphological, physiological or ecological traits (Moran 1994) are widespread in diverse groups such as amphibians, crustaceans, insects, and molluscs. Often, these stages are separated by a niche shift (Rowe and Ludwig 1991), for example, the larva occurs in an aquatic habitat and the adult inhabits a terrestrial habitat such as in many amphibians and aquatic insects. Two major sources of variation in timing of transitions between stages have been proposed: predation risk (the risk of being preyed upon) and time constraints (limits imposed by seasonality). Empirical and theoretical studies have shown predatorinduced plasticity, i.e., changes in the timing of and size at the stage transition, traits considered to be critical for adult fitness, under predation risk. Because foraging can increase encounters and detection by predators and hence predation risk (Stoks et al. 2005b), it is often decreased in the presence of predators resulting in a delayed transition to the adult stage at a smaller size.

Limited time constrains life histories (Rowe and Ludwig 1991, Abrams et al. 1996). Common time constraints include the availability of food for nestlings that determines breeding dates in birds (Rowe et al. 1994)

Manuscript received 8 September 2005; revised 22 November 2005; accepted 14 December 2005. Corresponding Editor: M. Wikelski.

${ }^{4}$ Corresponding author; E-mail: jor@sheffield.ac.uk and the necessity to reach sexual maturity in the reproductive season (Johansson et al. 2001). In line with model predictions, development time typically decreases under time constraints (e.g., Johansson et al. 2001, De Block and Stoks 2005). Thus, predation risk and time constraints represent antagonistic pressures on development time.

Theoretical work predicts that predation risk and time constraints should jointly shape life history, with prey animals being less responsive to predation risk when under time constraints (Rowe and Ludwig 1991, Werner and Anholt 1993, Abrams et al. 1996). Animals under a time constraint should prioritize fast development before the onset of an upcoming unfavorable period (e.g., winter or pond drying) over the risk of mortality by predation when the latter is smaller than the risk of death during the approaching unfavorable period. To date, very few studies have looked at the consequences of combined long-term exposure to predators and time constraints. The findings of these studies were largely consistent with the prediction that time constraints reduce the responsiveness to predators (Laurila and Kujasalo 1999, Johansson et al. 2001, Altwegg 2002), but focused on life history traits only.

Physiological traits have largely been neglected in studies evaluating the impact of environmental factors on life histories. However, recent studies suggest that traits such as investment in both immune response and in energy storage may also be important variables to consider (Moret and Schmid-Hempel 2000, Zera and 


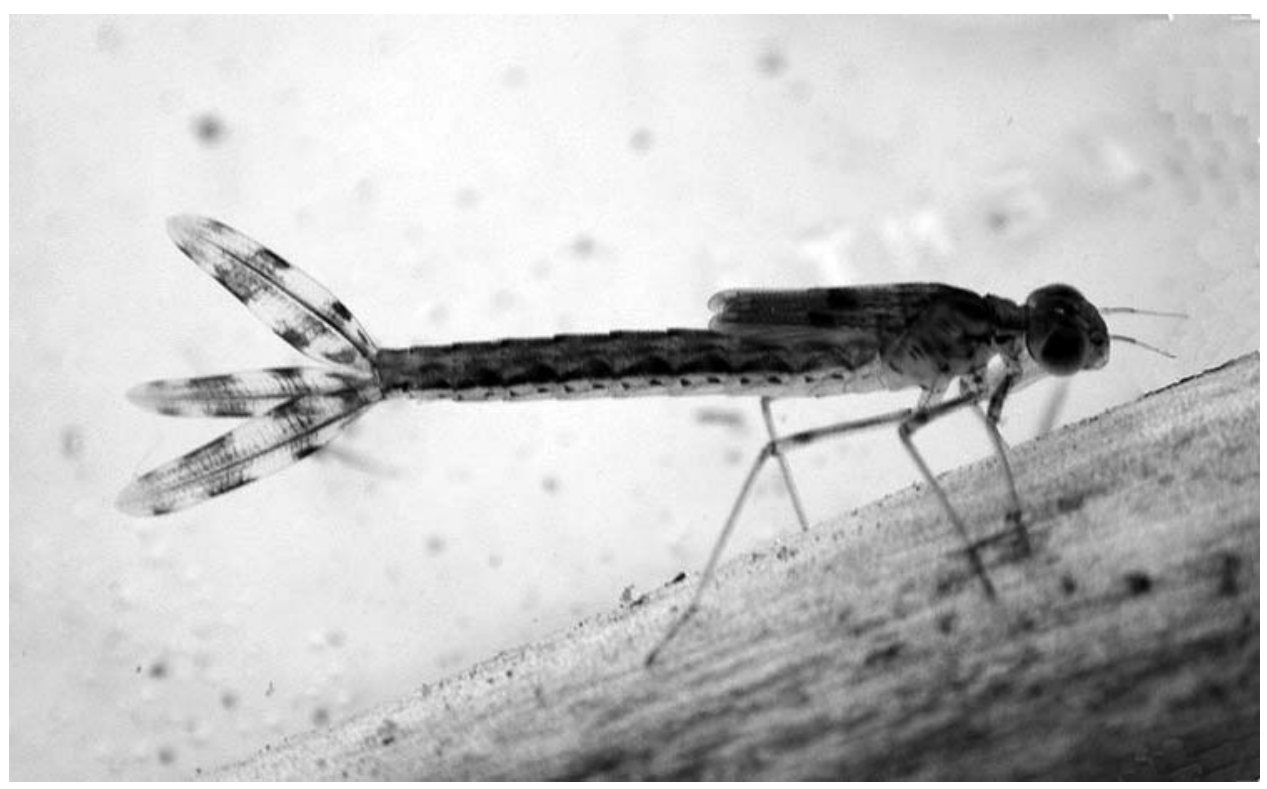

Plate 1. Larva of the damselfly Lestes viridis, a univoltine species known to react strongly to time contraints. Photo credit: F. Van de Meutter.

Harshman 2002). First, both immune function (Boonstra et al. 1998, Rigby and Jokela 2000, Zhang et al. 2003; but see Joop and Rolff 2004) and energy storage can be negatively affected by predation risk (Scheuerlein et al. 2001, Stoks et al. 2005a) and time constraints (Rolff et al. 2004). Second, physiological traits may shape adult fitness (Rolff and Siva-Jothy 2003, 2004). Finally, there is some evidence that physiological variables show responses to environmental factors independent of traditional life history variables (Rolff et al. 2004).

Here, we evaluate whether two major factors to understand the life histories of organisms with complex life cycles, predation risk and time constraints (Rowe and Ludwig 1991, Abrams et al. 1996, Benard 2004), also jointly shape the plasticity of physiological traits, immune function and condition, and whether these physiological traits are affected independently of traditional life history traits. We used the damselfly Lestes viridis (see Plate 1) as a study species and fish as predators, as these are very efficient predators on Lestes larvae (Stoks and De Block 2000), leaving no wounded survivors. Therefore, we do not expect any prophylactic increase in immune defense under predation risk to anticipate wounding (cf. Joop and Rolff 2004). The component of the immune system we studied is the phenoloxidase (PO) cascade. PO is involved in clearance of many different pathogens (Braun et al. 1998) as well as in wound repair (Sugumaran 2002). There is good evidence from damselflies that a higher PO activity confers resistance against gregarine parasites (Siva-Jothy 2000). The component of energy we study is fat (Rolff and Joop 2002, Stoks et al. 2005a). Fat storage is known to be important for starvation resistance and is linked to mating success and survival when parasitized (Plaistow and Siva-Jothy 1996). Based on previous empirical and theoretical work we predict that (1) under time constraints, larvae will emerge earlier at a smaller mass with lower fat storage and lower PO activity; (2) under predation risk, larvae will emerge later at a smaller mass with lower fat storage and a lower PO activity; (3) these predator-induced changes in life history and physiology will be smaller under time constraints; and (4) physiological responses to predation risk and time constraints will be independent of traditional life history responses.

\section{Methods}

\section{Experimental setup}

One-day old larvae were collected in Wilrijk (Belgium) using the technique described in De Block et al. (2005). Early-hatched larvae were collected on 22 April 2004, late-hatched larvae on 12 May (De Block et al. 2005). For each date, larvae were randomly allocated to one of the four combinations of manipulated time constraint and predation risk. We imposed a time constraint by manipulating photoperiod (Johansson and Rowe 1999). Damselflies commonly use day length as a developmental cue (Corbet 1999). This gave a full-factorial randomized design with two levels of hatching date (early vs. late), photoperiod (actual vs. delayed), and predation risk (present vs. absent). Larvae were reared in climate rooms (mean, $22^{\circ} \mathrm{C}$; range, $21-23^{\circ} \mathrm{C}$ ) at the photoperiod corresponding with the actual date (actual photoperiod; this started at 20 April with a light:dark ratio of 14:10 for the early-hatched larvae and at 10 May with a light:dark ratio of 15.5:8.5 for the late-hatched larvae) or at the photoperiod larvae would experience if eggs had hatched six weeks later (delayed photoperiod; 
this started at 1 June with a light:dark ratio of 16:8 for the early-hatched larvae and at 21 June with a light:dark ratio of $16.5: 7.5$ for the late-hatched larvae). Throughout the experiment, photoperiods were adjusted every 10 days to simulate the natural progress of the light cycle. Larvae and their respective light condition were rotated between climate rooms every 10 days.

Predation risk was manipulated by exposing half of the larvae to a combination of four predator stimuli: visual cues of a predatory fish and predatory conspecifics as well as fish kairomones and larval alarm chemicals (Chivers et al. 1996). Larvae were reared individually in plastic cups (diameter, $5 \mathrm{~cm}$; height, $9 \mathrm{~cm}$ ) filled to a height of $6 \mathrm{~cm}$ with filtered pond water. Six opaque cups and six transparent cups were placed on opposite sides at the bottom of a $30-\mathrm{L}$ aquarium $(47.5 \times 22.5 \times 26.5 \mathrm{~cm})$ filled to a height of $5 \mathrm{~cm}$ with aged tap water. The aquarium was divided in two halves with a metal frame and one stickleback (Gasterosteus aculeatus, standard length $5 \mathrm{~cm}$ ) was added to the side with transparent cups. Larvae in these transparent cups could not only see each other (Lestes damselfly larvae are cannibalistic [De Block and Stoks 2004a]) but also the stickleback predator. Moreover, together with food, we gave these larvae $500 \mu \mathrm{L}$ of fish medium combined with one crushed larva; larvae reared without predation risk were given $500 \mu \mathrm{L}$ of aged tap water. Fish medium was prepared by keeping two fish in a $30-\mathrm{L}$ aquarium, which was filled to a depth of $5 \mathrm{~cm}$ with aged tap water. Each fish was fed one L. viridis larva daily, $2 \mathrm{~h}$ prior to adding the fish medium to the experimental larvae. This way the fish medium contained both fish kairomones and damselfly alarm chemicals (Chivers et al. 1996). When larvae were 30 d old, we exposed them to a larger fish predator: pumpkinseed (Lepomis gibbosus, standard length $8 \mathrm{~cm}$ ). Therefore, we raised the water level in the aquaria to $9 \mathrm{~cm}$. A metal frame with 12 openings was placed in each aquarium. In each opening, we placed one cup (the six transparent ones at the side with the fish and the six white cups at the side without fish), which was submersed to a depth of $6 \mathrm{~cm}$. There were 20 aquaria for each combination of photoperiod and hatching date $(n=80$ aquaria); giving a total of 120 larvae at each treatment combination ( $n=960$ larvae). Each larva was fed laboratory-reared Artemia nauplii daily. From hatching until they entered the final instar, the mean food portion fed to a single larva on each feeding occasion contained 311 shrimps $(\mathrm{SE}=17, n=5)$. This corresponds with a high food level (Johansson et al. 2001). These nauplii were dissolved in $500 \mu \mathrm{L}$ of aged tap water (fish-free treatment), or in $500 \mu \mathrm{L}$ of fish medium (fish treatment). After molt into the final instar, we switched to a daily food ration of two Chironomus larvae. We increased food ration as larger larvae need more food (as in Johansson et al. 2001).

\section{Response variables}

Larval development time is taken as the number of days from egg hatching until emergence. Size at emergence was measured as dry mass. We analyzed the fat content of the abdomen and thorax following the protocol of Plaistow and Siva-Jothy (1996). They were dried for $24 \mathrm{~h}$ to constant mass in a drying oven $\left(80^{\circ} \mathrm{C}\right)$ and subsequently weighed to the nearest $0.01 \mathrm{mg}$ (Mettler balance; Mettler-Toledo, Leicester, UK). The fat was extracted in a Soxhlett apparatus (Schott AG, Mainz, Germany) by chloroform reflux for $8 \mathrm{~h}$. Thereafter, the abdomens and thoraxes were dried and reweighed. Fat content was calculated as dry mass before minus dry mass after extraction.

The phenoloxidase (PO) cascade is an important player in insect immune function (Sugumaran 2002). Its inactive form is stored in hemocytes as prophenoloxidase (proPO). To analyze PO-activity hemolymph samples were obtained by perfusing with $0.3 \mathrm{~mL}$ cacodylate buffer $(0.01 \mathrm{~mol} / \mathrm{L} \mathrm{Na}-\mathrm{Coc}, 0.005 \mathrm{~mol} / \mathrm{L}$ $\mathrm{CaCl}_{2}$ [syringe: Micro-fine, $0.5 \mathrm{~mL}, 0.33 \times 12.7 \mathrm{~mm}$, Beckton Dickinson, Erembodegem, Belgium]). The hemolymph extract was collected in $1.5-\mathrm{mL}$ microcentrifuge tubes and the samples were immediately frozen at $-80^{\circ} \mathrm{C}$. For the spectrophotometric assay, the samples were thawed in ice water. The cell walls were removed via centrifugation $\left(4^{\circ} \mathrm{C}, 6500 \mathrm{rpm}, 15 \mathrm{~min}\right.$, Eppendorf centrifuge 5417R [Eppindorf UK, Cambridge, UK]). Twenty microliters of the supernatant was added to $20 \mu \mathrm{L}$ L-DOPA $(10 \mathrm{mmol} / \mathrm{L}$ in cacodylate buffer), $20 \mu \mathrm{L}$ PBS, and $140 \mu \mathrm{L}$ distilled water. The reaction was allowed to proceed for $30 \mathrm{~min}$ at $30^{\circ} \mathrm{C}$. During the reaction, the enzyme catalyses the transition from L-Dopa to dopachrome (Sugumaran 2002). Dopachrome can than be measured photometrically. Readings were taken every $10 \mathrm{~s}$ on a plate reader (Versamax, Molecular Devices, Sunnyvale, California, USA). Enzyme activity was measured as $V_{\max }$ (the slope of the reaction curve during the linear phase).

We measured proPO following a modified protocol by Jacot et al. (2005). ProPO was activated using chymotrypsin. Reaction mixtures contained $50 \mu \mathrm{L}$ L-Dopa (12 $\mathrm{mg} / 10 \mathrm{~mL}$ aqua dest), $20 \mu \mathrm{L}$ PBS, $5 \mu \mathrm{L}$ chymotrypsin (5 $\mathrm{mg} / 1 \mathrm{~mL}$ aqua dest), $105 \mu \mathrm{L}$ distilled water, and $20 \mu \mathrm{L}$ sample. The mixture was incubated in a 96-well plate for five minutes at room temperature. $V_{\max }$ was determined as for PO.

\section{Statistical analyses}

We tested for effects of hatching date, photoperiod, and predation risk on survival in a repeated-measures ANOVA with hatching date and photoperiod as independent variables and the number of survived larvae reared with and without predation risk in each aquarium as the repeats. We did not include sex as several larvae died before they could be sexed. We tested for effects of hatching date, photoperiod, predation risk, and sex on the life history traits development time and mass at emergence in separate mixed-model ANOVAs using PROC MIXED in SAS 9.1 (Brown and Prescott 1999, SAS Institute 2004). Aquarium was defined as a random variable and nested in the hatching date by 

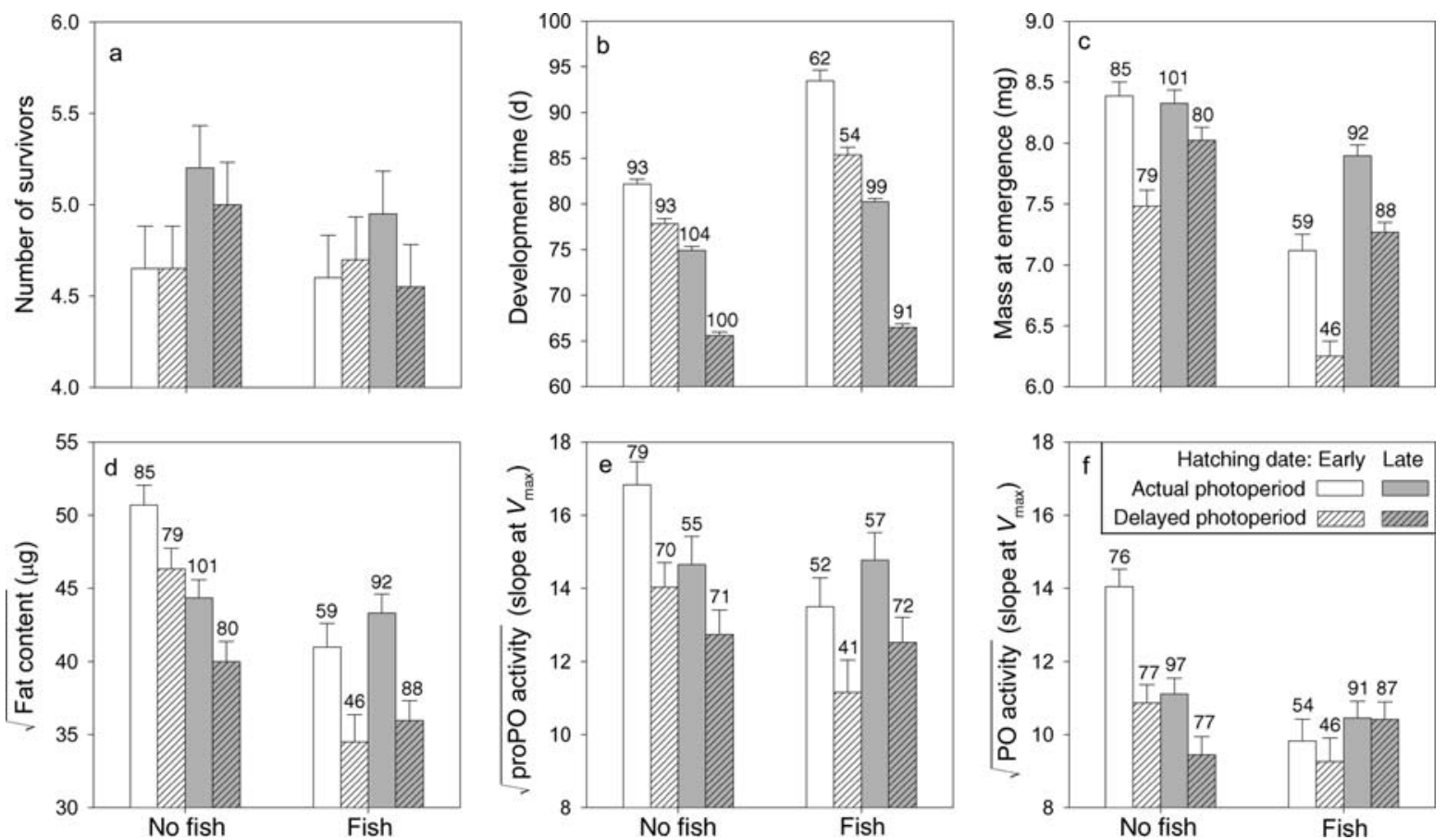

FIG. 1. Life history traits (a) number of survivors per aquarium side (out of six), (b) development time, and (c) mass at emergence, and the physiological variables (d) fat content, (e) proPO (prophenoloxidase) activity, and (f) PO (phenoloxidase) activity of Lestes viridis larvae as a function of hatching date, photoperiod, and predation risk. Values are means $+\mathrm{SE}$; least-squares means are given for the physiological variables, corrected for development time and mass at emergence. Numbers above the bars represent the sample sizes; for (a), means are based on 20 aquaria. Photoperiods at hatching (reflecting proximity to winter) for the different time constraint categories correspond with following dates: 20 April (early hatched, actual photoperiod), 10 May (late hatched, actual photoperiod), 1 June (early hatched, delayed photoperiod), and 21 June (late hatched, delayed photoperiod). Enzyme activity was measured at $V_{\max }$ (maximum velocity, the slope of the reaction curve during the linear phase).

photoperiod interaction; also interactions with aquarium were included in the model. This allowed us to take advantage of the full data set while overcoming the problem with pseudoreplication within aquaria (see Brown and Prescott 1999, Millar and Anderson 2004). As sex was not a focal variable we only include sex as a main effect. Effects of hatching date, photoperiod and predation risk on the physiological variables (fat content, proPO activity, PO activity) were tested with development time and mass at emergence as covariates in separate ANCOVAs. Interactions between treatments and the covariates were not significant and dropped from final analyses. We started with models including all higher order interactions between the factors and proceeded with stepwise simplification of the models removing the highest order interaction term that was not

TABLE 1. Results from $\mathrm{AN}(\mathrm{C}) \mathrm{OVAs}$ testing for effects of hatching date, photoperiod, and predation risk on life history variables (development time and mass) and physiological variables (fat content, proPO [prophenoloxidase] activity, and PO [phenoloxidase] activity).

\begin{tabular}{|c|c|c|c|c|c|c|c|c|c|c|}
\hline \multirow[b]{2}{*}{ Factors } & \multicolumn{2}{|c|}{ Development time } & \multicolumn{2}{|c|}{ Mass } & \multicolumn{2}{|c|}{ Fat content } & \multicolumn{2}{|c|}{ ProPO activity } & \multicolumn{2}{|c|}{ PO activity } \\
\hline & $\mathrm{df}$ & $F$ & df & $F$ & df & $F$ & $\mathrm{df}$ & $F$ & $\mathrm{df}$ & $F$ \\
\hline Hatching date $(\mathrm{H})$ & $1,71.7$ & $322.56^{* * *}$ & $1,97.9$ & $38.01 * * *$ & 1,272 & $5.62 *$ & 1,262 & 0.00 & 1,237 & $5.74 *$ \\
\hline Photoperiod (Ph) & $1,71.6$ & $129.30 * * *$ & $1,95.1$ & $49.54 * * *$ & 1,223 & $5.63^{*}$ & 1,187 & 0.94 & 1,201 & 3.55 \\
\hline Predation risk $(\mathrm{Pr})$ & $1,68.6$ & $171.84 * * *$ & 1,116 & $114.31 * * *$ & 1,590 & $4.86^{*}$ & 1,222 & 2.69 & 1,222 & 2.36 \\
\hline $\mathrm{H} \times \mathrm{Ph}$ & $1,71.8$ & $15.56^{* * *}$ & $1,95.7$ & $5.74 *$ & NS & NS & NS & NS & NS & NS \\
\hline $\mathrm{H} \times \operatorname{Pr}$ & $1,68.4$ & $40.05^{* * *}$ & 1,116 & $18.72 * * *$ & 1,588 & $5.31^{*}$ & 1,169 & $6.86^{* *}$ & 1,161 & $10.00 * *$ \\
\hline $\mathrm{Ph} \times \operatorname{Pr}$ & $1,66.5$ & $24.25^{* * *}$ & NS & NS & NS & NS & NS & NS & 1,137 & $4.16^{*}$ \\
\hline $\mathrm{H} \times \mathrm{Ph} \times \mathrm{Pr}$. & NS & NS & NS & NS & NS & NS & NS & NS & NS & NS \\
\hline Development time & & & & & 1,443 & 0.14 & 1,329 & 2.39 & 1,400 & 0.29 \\
\hline Mass & & & & & 1,560 & $64.33 * * *$ & 1,452 & $15.28 * * *$ & 1,558 & $5.51^{*}$ \\
\hline
\end{tabular}

Notes: All analyses included sex; for the physiological variables, both development time and mass were included as covariates. Nonsignificant interactions were removed and indicated by NS.

$* P \leq 0.05 ; * * P \leq 0.01 ; * * * P \leq 0.001$. 
significant first (Crawley 2002). Physiological variables were square-root-transformed to meet ANCOVA assumptions. Nine measurements of proPO activity and 14 of PO activity were more than three standard deviations from the mean and considered as outliers and not included in the analyses. Because of mortality, unsuccessful emergences and loss of a subset of the samples and the sex information on some adults, the final design was unbalanced. Therefore, we used the Satterthwaite formula as implemented in SAS to obtain correct degrees of freedom. Moreover, the Satterthwaite correction is recommended if the model contains different error strata (Brown and Prescott 1999), as in our nested model.

\section{RESULTS}

Life history.-Overall survival from the egg stage onward was $80 \%$ and was not affected by treatments (repeated-measures ANOVA; all $P>0.10$; Fig. 1a). Development time was shorter in late-hatched larvae than in early-hatched larvae, and shorter at the delayed photoperiod than at the actual photoperiod, and these effects strengthened each other (hatching date $\times$ photoperiod, Table 1, Fig. 1b). Development time was longer in larvae reared in the presence of the fish predators than when the fish was absent (Table 1, Fig. 1b). This effect of predation risk was smaller in late-hatched larvae than in early-hatched larvae (hatching date $\times$ predation risk), and smaller at the delayed photoperiod than at the actual photoperiod (photoperiod $\times$ predation risk, Table 1).

Overall, mass at emergence was lower at the delayed photoperiod than at the actual photoperiod (Table 1, Fig.1c). This photoperiod effect was more pronounced in early-hatched larvae (hatching date $\times$ photoperiod). Mass was reduced under predation risk, but less so in late-hatched larvae (hatching date $\times$ predation risk, Table 1).

Physiology.-Fat content was lower at the delayed photoperiod than at the actual photoperiod (Table 1, Fig. 1d). Fat content was reduced under predation risk, but less so in late-hatched larvae (hatching date $\times$ predation risk, Table 1). The presence of the fish predator reduced $\mathrm{PO}$ and proPO activity in earlyhatched larvae, but not in late-hatched larvae (hatching date $\times$ predation risk; Table 1, Fig. 1e-f). Similarly, predation risk decreased PO activity at the actual photoperiod, but not at the delayed photoperiod (photoperiod $\times$ predation risk).

\section{Discussion}

Our results show that both predation risk and time constraints affected life history and physiology. Moreover, time constraints shaped the predator-induced plasticity in life history and physiology, albeit in different ways. Overall, larvae accelerated development and growth under both natural (hatching date) and manipulated (photoperiod) time constraints as predicted by theory (e.g., Rowe and Ludwig 1991, Abrams et al. 1996) and as shown in previous studies in this species (De Block and Stoks 2004b, 2005). Under predation risk, larvae slowed development and growth and emerged at a smaller mass as predicted by theory when animals show flexibility in growth effort (e.g., foraging rate), size and age at metamorphosis (Abrams and Rowe 1996). These effects of predation risk on life history were less pronounced in late-hatched larvae than in early-hatched larvae, and for development also less pronounced at the delayed than at the actual photoperiod. Such decreased responsiveness to predation risk under time constraints has been predicted by several models (Rowe and Ludwig 1991, Werner and Anholt 1993, Abrams et al. 1996). Time-constrained animals should prioritize fast development before the onset of an approaching unfavorable period (e.g., winter or pond drying) over the risk of mortality by predation when the latter is smaller than the risk of death during the upcoming unfavorable period. This prediction holds for our study species as larvae cannot survive either the winter or pond drying (Jödicke 1997). Similarly, larvae of the damselfly Lestes sponsa reared at a delayed photoperiod showed a smaller responsiveness to a predator with regard to mass at emergence (Johansson et al. 2001), and growth rate (Stoks et al. 2005b). Also, the only two other studies that simultaneously considered time and predation constraints on larval life history from the egg stage, both on tadpoles, reported similar effects of time constraints on predator-induced life history plasticity (Laurila and Kujasalo 1999, Altwegg 2002).

Time constraints had negative effects on fat storage and immune function. This result is consistent with the observed higher growth rates under time constraints and the assumed trade-offs between growth rate and the costly investment in energy storage and immune response (Gotthard 2001). A reduction in fat content under time constraints has been demonstrated before in this species (Stoks et al. 2006) and in Calopteryx damselflies (Plaistow and Siva-Jothy 1999). In another study on L. viridis, no reduction in fat content under a delayed photoperiod was shown (Rolff et al. 2004). However, Rolff et al. (2004) reared damselflies at a different food regime and at a lower temperature than the current study, which might explain the differences in findings.

We show here, for the first time, that time constraints modulate the predator-induced plasticity in physiological traits. Time constrained larvae are probably closer to some minimum level of energy storage and immune function necessary to sustain further development; therefore they cannot exhibit as much plasticity. This conjecture may explain why physiological traits were reduced less (fat content) or not at all (proPO and PO activity) in late-hatched larvae under predation risk. Reduced investment in energy storage under predation risk has been shown before in another damselfly (Stoks et al. 2005b) and in fish (Pratt and Fox 2002, Garvey et 
al. 2004). Reduced immune function under predation risk has been demonstrated in a few other studies (Boonstra et al. 1998, Rigby and Jokela 2000, Zhang et al. 2003; but see Joop and Rolff 2004). This illustrates that predation risk may affect how the allocation of limiting resources are prioritized by potential prey. Investment in energy storage and immune function should receive less priority when prey organisms are exposed to predators because of the costs associated with these investments relative to the benefit of investing in reproductive life span at this stage.

Our data clearly indicate that predation risk and time constraints affected physiological traits independently of traditional life history variables such as age and mass at emergence. This perspective advocates studying these physiological traits since fat content and immune response are important physiological underpinnings of survival and reproductive success in damselflies (Plaistow and Siva-Jothy 1996, Sokolovska et al. 2000, Rolff and Siva-Jothy 2004). We are currently exploring to what extent studies of the impact of these environmental factors that focus solely on traditional life history traits and ignore physiological traits underestimate fitness costs in the adult stage.

We have also shown that the sublethal impact of predation on life history and physiology may differ strongly depending on the perceived time constraints. Time constraints, which are largely ignored in studies of predator-induced plasticity, may therefore explain some of the inconsistencies in findings from plasticity studies. In a recent review of predator-induced plasticity (Benard 2004), a plea was made to consider the potential interplay between plasticity in life history, behavior and morphology to gain further insight into variation in metamorphic responses. Our results suggest that physiological traits are also important components of metamorphic responses, and that time constraints should be considered in order to better understand predator-induced life history plasticity.

\section{ACKNOWLEDGMENTS}

We are grateful to Andrew Beckerman (again), Helen Crudgington, and four anonymous referees whose comments improved the paper and to Jonathan Ward and Rhiannon Pursall for help in the laboratory. J. Rolff is a Natural Environmental Research Council Fellow, M. De Block and R. Stoks are postdoctoral fellows at the Fund for Scientific Research Flanders (FWO), and S. Slos and W. van Doorslaer are Ph.D. scholars of the Institute for the Promotion of Innovation through Sciences and Technology in Flanders (IWT-Vlaanderen). This work was financed by FWO grant G.0269.04, and research grant OT/04/23 of the KULeuven Research Fund.

\section{Literature Cited}

Abrams, P. A., O. Leimar, S. Nylin, and C. Wiklund. 1996. The effect of flexible growth rates on optimal sizes and development times in a seasonal environment. American Naturalist 147:381-395.

Abrams, P. A., and L. Rowe. 1996. The effects of predation on the age and size of maturity of prey. Evolution 50:10521061.
Altwegg, R. 2002. Predator-induced life-history plasticity under time constraints in pool frogs. Ecology 83:2542-2551.

Benard, M. F. 2004. Predator-induced phenotypic plasticity in organisms with complex life histories. Annual Reviews of Ecology and Systematics 35:651-673.

Boonstra, R., D. Hik, G. R. Singleton, and A. Tinnikov. 1998. The impact of predator-induced stress on the snowshoe hare cycle. Ecological Monographs 68:371-394.

Braun, A., J. A. Hoffmann, and M. Meister. 1998. Analysis of the Drosophila host defense in domino mutant larvae, which are devoid of hemocytes. Proceedings of the National Academy of Sciences (USA) 95:14337-14342.

Brown, H., and R. Prescott. 1999. Applied mixed models in medicine. Wiley, Chichester, UK.

Chivers, D. P., B. D. Wisenden, and R. J. F. Smith. 1996 Damselfly larvae learn to recognize predators from chemical cues in the predator's diet. Animal Behaviour 52:315-320.

Corbet, P. S. 1999. Dragonflies. Behaviour and ecology of Odonata. Harley Books, Colchester, UK.

Crawley, M. J. 2002. Statistical computing. John Wiley and Sons, Chichester, UK.

De Block, M., and R. Stoks. 2004a. Cannibalism-mediated life history plasticity to combined time and food stress. Oikos 106:587-597.

De Block, M., and R. Stoks. 2004b. Life-history variation in relation to time constraints in a damselfly. Oecologia 140:6875.

De Block, M., and R. Stoks. 2005. Fitness effects from egg to reproduction: bridging the life history transition. Ecology 86: 185-197.

De Block, M., R. Stoks, and L. De Bruyn. 2005. Egg hatching patterns within and among populations of a damselfly occupying permanent and temporary ponds. Archiv für Hydrobiologie 163:195-209.

Garvey, J. E., K. G. Ostrand, and D. H. Wahl. 2004. Energetics, predation, and ration affect size-dependent growth and mortality of fish during winter. Ecology 85: 2860-2871.

Gotthard, K. 2001. Growth strategies of ectothermic animals in temperate environments. Pages 287-303 in D. Atkinson and M. Thorndyke, editors. Environment and animal development: genes, life histories and plasticity. BIOS, Oxford, UK.

Jacot, A., H. Scheuber, J. Kurtz, and M. W. G. Brinkhof. 2005. Juvenile immune system activation induces a costly upregulation of adult immunity in field crickets Gryllus campestris. Proceedings of the Royal Society London B 272: 63-69.

Jödicke, R. 1997. Die Binsenjungfern und Winterlibellen Europas. Westarp, Magdeburg, Germany.

Johansson, F., and L. Rowe. 1999. Life history theory and behavioral responses to time constraints in a damselfly. Ecology 80:1242-1252.

Johansson, F., R. Stoks, L. Rowe, and M. De Block. 2001. Life history plasticity in a damselfly: effects of combined time and biotic constraints. Ecology 82:1857-1869.

Joop, G., and J. Rolff. 2004. Plasticity of immune function and condition under the risk of predation and parasitism. Evolutionary Ecology Research 6:1051-1062.

Laurila, A., and J. Kujasalo. 1999. Habitat duration, predation risk and phenotypic plasticity in common frog (Rana temporaria) tadpoles. Journal of Animal Ecology 68:11231132.

Millar, R. B., and M. J. Anderson. 2004. Remedies for pseudoreplication. Fisheries Research 70:397-407.

Moran, N. A. 1994. Adaptation and constraint in the complex life-cycles of animals. Annual Review of Ecology and Systematics 25:573-600.

Moret, Y., and P. Schmid-Hempel. 2000. Survival for immunity: the price of immune system activation for bumblebee workers. Science 290:1166-1168. 
Plaistow, S., and M. T. Siva-Jothy. 1996. Energetic constraints and male mate-securing tactics in the damselfly Calopteryx splendens xanthostoma (Charpentier). Proceedings of the Royal Society London B 263:1233-1239.

Plaistow, S., and M. T. Siva-Jothy. 1999. The ontogenetic switch between odonate life history stages: effects on fitness when time and food are limited. Animal Behaviour 58:659667.

Pratt, T. C., and M. G. Fox. 2002. Influence of predation risk on the overwinter mortality and energetic relationships of young-of-year walleyes. Transactions of the American Fisheries Society 131:885-898.

Rigby, M. C., and J. Jokela. 2000. Predator avoidance and immune defence: costs and trade-offs in snails. Proceedings of the Royal Society London B 267:171-176.

Rolff, J., and G. Joop. 2002. Estimating condition: pitfalls of using weight as a fitness correlate. Evolutionary Ecology Research 4:931-935.

Rolff, J., and M. T. Siva-Jothy. 2003. Invertebrate ecological immunology. Science 301:472-475.

Rolff, J., and M. T. Siva-Jothy. 2004. Selection on insect immunity in the wild. Proceedings of the Royal Society of London B 271:2157-2160.

Rolff, J., F. Van de Meutter, and R. Stoks. 2004. Time constraints decouple age and size at maturity and physiological traits. American Naturalist 164:559-565.

Rowe, L., and D. Ludwig. 1991. Size and timing of metamorphosis in complex life cycles: time constraints and variation. Ecology 72:413-427.

Rowe, L., D. Ludwig, and D. Schluter. 1994. Time, condition, and the seasonal decline of avian clutch size. American Naturalist 143:698-772.

SAS Institute. 2004. SAS version 9.1. SAS Institute, Cary, North Carolina, USA.

Scheuerlein, A., T. J. Van't Hof, and E. Gwinner. 2001. Predators as stressors? Physiological and reproductive consequences of predation risk in tropical stonechats (Saxicola torquata axillaris). Proceedings of the Royal Society of London B 268:1575-1582.

Siva-Jothy, M. T. 2000. A mechanistic link between parasite resistance and expression of a sexually selected trait in a damselfly. Proceedings of the Royal Society of London B 267:2523-2527.

Sokolovska, N., L. Rowe, and F. Johansson. 2000. Fitness and body size in mature odonates. Ecological Entomology 25: 239-248.

Stoks, R., and M. De Block. 2000. The influence of predator species and prey age on the immediate survival value of antipredator behaviours in a damselfly. Archiv für Hydrobiologie 147:417-430.

Stoks, R., M. De Block, and M. A. McPeek. 2005a. Alternative growth and energy storage responses to mortality threats in damselflies. Ecology Letters 8:1307-1316.

Stoks, R., M. De Block, and M. A. McPeek. 2006. Physiological costs of compensatory growth in a damselfly. Ecology, in press.

Stoks, R., M. De Block, F. Van de Meutter, and F. Johansson. $2005 \mathrm{~b}$. Predation cost of rapid growth: behavioural coupling and physiological decoupling. Journal of Animal Ecology 74: 708-715.

Sugumaran, H. 2002. Comparative biochemistry of eumelanogenesis and the protective roles of phenoloxidase and melanin in insects. Pigment Cell Research 15:2-9.

Werner, E. E., and B. R. Anholt. 1993. Ecological consequences of the trade-off between growth and mortality-rates mediated by foraging activity. American Naturalist 142:242-272.

Zera, A. J., and L. G. Harshman. 2002. The physiology of life history trade-offs in animals. Annual Review of Ecology and Systematics 32:95-126.

Zhang, J. X., C. Cao, H. Gao, Z. S. Yang, L. X. Sun, Z. B. Zhang, and Z. W. Wang. 2003. Effects of weasel odor on behavior and physiology of two hamster species. Physiology and Behavior 79:549-552. 